\title{
LOS PROFESIONALES DE TRABAJO SOCIAL ADMINISTRADORES EN LA ERA DE LA GLOBALIZACIÓN
}

Lourdes I. Morales Alejandro ${ }^{1}$

\section{Resumen}

Las instituciones sociales públicas y privadas, así como la vida económica, social, política y moral de los países se enfrentan a una serie de cambios producto del surgimiento de la sociedad post industrial caracterizada, por entre otras cosas, por la globalización mundial, por una economía de consumo, producción de información, avances científico-tecnológicos, la investigación, el respeto y aceptación de la diversidad cultural, la autonomía y la comunidad como entidad social. La globalización de la economía mundial y el aumento de los movimientos sociales proponen nuevos retos y oportunidades a profesionales de trabajo social en puestos de administración. La transformación que sufre el sistema de bienestar social conduce a nuevos escenarios de cultura gerencial y una redefinición de la administración y supervisión. Profesionales en estos puestos deben tener capacidad para integrar sus conocimientos teórico-científicos en el proceso de reunir e integrar todos los esfuerzos institucionales con múltiples propósitos en forma deliberada, por lo que las destrezas del trabajo multidisciplinario y en equipo es altamente valorado. Ante el reto de las contribuciones y amenazas de la globalización mundial y su impacto en la práctica, deben considerar herramientas de acción para convertirse en el puente entre lo local y lo global.

Descriptores: administración en trabajo social, globalización, trabajo social.

\section{Abstract}

Private and public organizations as well the economic, social, moral and political nation's life confront serious changes as a result of the post industrial society, characterized, among other things, by the world globalization, economy of consume, production of information, scientific and technological innovations, research, the respect and acceptation of cultural

\footnotetext{
${ }^{1}$ Profesora en el Departamento de Trabajo Social, Facultad de Ciencias Sociales, Universidad de Puerto Rico en Río Piedras.
} 
diversity and the community as a social entity. The globalization of the world economy and ever-growing social movements propose new opportunities and challenges to social workers administrators. The transformation of the social welfare system leads to a new management culture and a redefinition of the supervision and administrative practice. These social workers administrators must have the ability to integrate their theoretical and scientific knowledge in a strategic and deliberate way to all the organizational process; because of this, their multidisciplinary and team work skills are highly valued. In front of the contributions and threats of the globalization and its impact to the social work practice, the social worker administrator must consider multiple and effective action tools in order to become a bridge between the local and global aspects.

Keywords: social work administration, globalization, social work.

\section{Introducción}

Las instituciones sociales públicas y privadas, así como la vida económica, social, política y moral de los países se enfrentan a una serie de cambios producto del surgimiento de la sociedad post industrial. Esta se caracteriza por una economía de consumo, una fuerza laboral orientada al servicio, a la producción de información, los avances científico-tecnológicos, la investigación, la globalización mundial, la democracia, el respeto y aceptación de la diversidad cultural, la autonomía y la comunidad como entidad social (Aponte, 1998; Concepción Wichy, 1998, citados en Morales Alejandro, 2001). Estos cambios pueden resumirse en tres áreas fundamentales: a) el surgimiento de la sociedad de información, que transforma la naturaleza del trabajo, b) la globalización mundial que afecta la economía y los aspectos sociopolíticos en el mundo y c) la revolución científico-tecnológica que afecta la ética y la moral (Yarzábal, 1997, citado en Morales Alejandro, 2001).

La globalización de la economía mundial y el aumento de los movimientos sociales proponen nuevos retos y oportunidades a los trabajadores sociales como administradores. Trabajar con las fuerzas de la globalización representa oportunidades, pero también riesgos, y pueden ser el motor que impulse el desarrollo de modelos de práctica que sean capaces de enfrentar los asuntos sociales que conllevan. El impacto y las respuestas a estos 
cambios se diferenciarán de acuerdo a los contextos culturales y políticos en que ocurran.

Ante el impacto de la globalización las estructuras sociales, políticas y económicas han cambiado. La expansión económica permite la comunicación sin precedentes, no sólo a través de los medios de comunicación, sino también a través de los intercambios culturales, económicos y destrezas, así también a través de la migración de la gente. Los aspectos negativos del impacto de la globalización se reflejan en la extracción de los recursos nacionales y la explotación de mano de obra barata, resultando esto en una gran disparidad económica entre el rico y el pobre. La movilidad de capital y los rápidos cambios económicos se presentan a los trabajadores y trabajadoras alrededor del mundo con cierres de plantas, disminución de personal, el disloque ocupacional y la pérdida de seguridad de empleo (Rowe, Handley, Repetur Moreno \& Mould, 2000). Las corporaciones multinacionales, con lealtades y responsabilidades a ningún país, están aumentando su manipulación de la economía global, finanzas y el gobierno. En la llamada economía global muchas naciones están limitadas en su capacidad para proveer programas de bienestar social y algunas personas aún cuestionan el valor de algunos de los objetivos que persiguen, que pueden ser realizados por un grupo pequeño de países relativamente acaudalados (Drover, 2000). El cada vez menor control sobre las condiciones económicas de las que dependen para financiar sus compromisos con la educación, salud, seguridad de ingresos y servicios sociales, representa fiscal y moralmente serias dificultades para responder a las demandas de bienestar de la sociedad.

Ante estos cambios mundiales y sociales las funciones y roles de las instituciones sociales están en plena transformación y el papel que desempeñan sus líderes está cambiando drásticamente. Estos cambios han traído alteraciones en las experiencias profesionales y capacidades personales que son requeridas en el liderazgo administrativo social. Nuevas demandas y retos enmarcan sus ejecutorias como profesional. Se espera que ante los retos que representan los cambios económicos, sociales y políticos mundiales a las instituciones sociales, este/a líder, no sólo aporte sus conocimientos, competencias y experiencias a la organización, sino también su compromiso con la pertinencia, calidad, prontitud de los servicios y con el desarrollo profesional de su personal, lo que redundará en la satisfacción de las necesidades de su clientela. La transformación que ha de sufrir el sistema de bienestar social conduce a nuevos escenarios de cultura gerencial, una 
redefinición de la administración y supervisión y al consiguiente impacto del proceso en la ayuda social.

\section{Trabajo social, administración y globalización}

La administración de agencias, como un método de trabajo social de servicio indirecto, ubica al profesional en trabajo social a la cabeza de una agencia, servicio o programa de bienestar social que se orienta hacia la dirección efectiva de esa organización, sea pública o privada, para garantizar una atención de calidad a las necesidades de las personas beneficiarias de dichos servicios o programas. Un administrador o administradora efectiva debe regirse por una serie de principios para la práctica de una sana administración, conforme a los valores de la profesión, las necesidades de los clientes, los objetivos de la agencia, el clima y cultura de la institución, la participación democrática, la comunicación, el liderazgo, la planificación, la organización, la delegación de funciones, la coordinación, el uso de recursos, el cambio, la evaluación y el crecimiento humano (Ruiz González, 1999). Es preocupante la cantidad de administradores de agencias sociales que no disponen del conocimiento o adiestramiento adecuado y formal para ejercer esta multiplicidad de responsabilidades y roles.

Profesionales de trabajo social en el ejercicio de sus responsabilidades y roles en puestos de administración, deben distinguirse por unas características y cualidades particulares. Estas características están sustancialmente compuestas por la combinación de dos dimensiones: a) la dimensión profesional, expresada a través del conocimiento, destrezas y talentos y b) la dimensión personal, expresada por su personalidad, sentimientos, valores y creencias personales. Sus capacidades administrativas requieren el conocimiento profundo de modelos, procesos y teorías administrativas que guíen su práctica (Ander- egg, 1986, citado en Caraballo, et al, 1992; Skidmore, 1995).

Profesionales de trabajo social en roles de administración que no posea los conocimientos y competencias administrativas actuará por intuición y la eficacia y efectividad de su práctica se verán afectadas y con ellas los servicios de la agencia. Los ejecutivos a los que responde, su personal, los clientes, la comunidad general y las agencias acreditadoras de los servicios que ofrecen, esperan que tenga control de los procesos administrativos y 
provea una dirección efectiva y eficaz a su agencia para el logro de las metas y objetivos propuestos en su misión. Este/a profesional debe tener capacidad para integrar sus conocimientos teórico-científicos en el proceso de reunir e integrar todos los esfuerzos institucionales con múltiples propósitos en forma deliberada, por lo que las destrezas colaborativas, el trabajo multidisciplinario y en equipo son altamente valorados.

Otras cualidades administrativas que deben poseer es la tolerancia al caos, las competencias necesarias para evaluar, enfrentarse a los riesgos y la capacidad de crear estrategias que vayan dirigidas a alcanzar sus metas. Ante el cambio, se recomienda absorber y contener la ansiedad del personal tanto durante el cambio o cuando las cosas no marchen bien. De este/a profesional se demandan destrezas administrativas o la habilidad para desarrollarlas en los procesos de toma de decisiones acerca de la gente a su cargo, los programas o los asuntos fiscales. Para tomar estas decisiones se requieren destrezas de comunicación, esto es, presteza para el diálogo, saber escuchar y discernir, reflexionar y actuar, ya sea mediando, manejando o solucionando conflictos. Su capacidad administrativa sobresale porque dirige, delega, trabaja, empodera (empowerment) a su personal para participar en la toma de decisiones y procura desarrollar a otros para que puedan asumir responsabilidades de liderazgo dentro y fuera de la institución.

El liderazgo de profesionales de trabajo social en puestos administrativos debe distinguirse por: a) una profunda comprensión de la interacción del sistema cliente y su ambiente, particularmente, la influencia de los movimientos sociales y mundiales en los sistemas de bienestar social; b) una conciencia global en la aceptación de la integración social y el compromiso con una práctica libre de prejuicios y por tanto, de discrimen; c) la creatividad en la innovación de modalidades de intervención; d) exhibir conocimientos y destrezas en investigación de manera que pueda realizar estudios sistemáticos sobre el estrés severo que están sufriendo los trabajadores desplazados y sus familias, tanto en el sufrimiento humano como en las pérdidas materiales; e) el compromiso con el desarrollo y preservación de un clima y cultura organizacional que mantenga la cohesión y espíritu de unidad en su personal; f) un liderazgo proactivo en el desarrollo de coaliciones que dirijan al diálogo abierto con los políticos a nivel regional, nacional e internacional que puedan ser usadas para un nuevo pensamiento y una nueva orientación en la transformación del bienestar social (Kaseke, 1998, citado en Rowe, Handley, Repetur Moreno \& Mould, 2000); g) la 
disposición de cooperar en esfuerzos existentes en su país para contrarrestar los impactos negativos de la globalización y tomar completa ventaja de sus oportunidades; h) un compromiso en el desarrollo e implantación de políticas y programas que redunden en el beneficio y éxito de su institución; i) demostrar conocimientos y destrezas organizativas para establecer prioridades en el desarrollo de procedimientos y procesos para el avalúo de sus programas y de su personal; j) su compromiso con el desarrollo de líderes en todos los niveles de su organización, centrados en principios y competencias en su quehacer; $\mathrm{k}$ ) la apertura y sensibilidad a las identidades multiculturales, a la diferencia y a la importancia del diálogo, demostrando una actitud de solidaridad, evidenciada por la responsabilidad por los demás, por la necesidad humana; 1) el ejercicio del rol de maestro y orientador, educando al personal acerca de las consecuencias de la reestructuración global y en conjunto buscar alternativas de acción; y m) aceptar el reto de ser promotor o promotora de una ciudadanía activa, en la cual las personas, las comunidades geográficas, ocupacionales, voluntarias y políticas se unan en un esfuerzo colaborativo y articulen una visión de la vida buena (Drover, 2000). Estas características se enmarcan en un liderazgo democrático, holístico, transformador y responsivo a los elementos culturales.

Por otro lado, la dimensión personal de la trabajadora y el trabajador social en administración se enmarca en sus características como persona, las que le posibilitarán laborar y enfrentar la multiplicidad y diversidad de retos que su puesto le exige. En el manejo de sí mismo/a y con otras personas, este/a profesional se espera sea consistente, tenga integridad, sea leal a los altos estándares de la profesión, tenga control de sus emociones, conozca sus sentimientos y esté en disposición de escuchar sugerencias. Se requiere un sistema de creencias que le permita la identificación con la visión de la agencia y a tomar $\mathrm{o}$ no en consideración el entorno inmediato, sus compañeros y compañeras de trabajo y su clientela. Su propia personalidad, moldeada por la crianza, experiencias de vida, espirituales, formación profesional, valores, compromisos y estilos de liderazgo, todos interactúan en el contexto institucional del escenario de trabajo. Se espera entonces que en su dimensión personal y espiritual, este/a profesional sea una persona equilibrada emocionalmente, sea inspiradora, de motivación y modelo para otros, que maneje efectivamente los conflictos profesionales y personales y que los niveles de estrés y conflicto no le impidan perder la sabiduría y el buen funcionamiento como líder administrativo. Estas características describen cualitativamente el desempeño y la multiplicidad de sus roles y a 
la misma vez sugieren un marco de referencia para entender, identificar y reclutar a los nuevos trabajadores sociales administradores.

La administración es una ciencia y un arte (Koontz \& Weihrich, 1998). Los conocimientos organizados constituyen una ciencia y en la práctica la administración es un arte, siendo ambos complementarios. Los conocimientos administrativos pueden mejorar la práctica de la administración. Las personas administradoras de agencias sociales que pretenden llevar a cabo sus funciones sin recurrir a la ciencia administrativa deben atenerse únicamente a la suerte, la intuición, a emular a otros o a lo que hicieron en el pasado. A menos que las personas practicantes de la administración como de cualquier otro campo, aprendan a fuerza de prueba y error, no encontrarán una orientación significativa en otra parte que no sea en el conocimiento acumulado subyacente en su práctica. La trabajadora o el trabajador social administrador/a ocupa diferentes puestos en la estructura organizativa de una institución, el más común es en la dirección de un departamento, puesto que de acuerdo a la literatura consultada, se conoce como gerente intermedio. Su responsabilidad es dirigir la implantación de las políticas de la agencia, las metas y objetivos y a la misma vez armonizar las demandas de sus superiores con las capacidades del personal a cargo (Stoner, 1986, citado en Caraballo, et al, 1992). A pesar de la importancia de sus responsabilidades y roles estos líderes poseen muy pocos ningún conocimiento y destrezas administrativas (Ander-egg, 1986 citado en Caraballo, et al, 1992), fenómeno que ocurre con frecuencia en Puerto Rico. Esta realidad lanza un reto a los programas académicos de las universidades que preparan a futuros profesionales de trabajo social. Es necesario que estos comprendan la importancia y necesidad de conocimientos y destrezas en esta ciencia y aumenten el número de horas o créditos académicos, complementándolos con la práctica. Deben además, desarrollar programas de certificación para profesionales que ya desempeñan estas funciones para prepararlos en esta materia. También es preciso enfatizar, que al acoger el código de ética como la guía por excelencia del desempeño profesional de todo trabajador y trabajadora social, se está haciendo un compromiso con ser aprendices autodirigidos y no utilizar como excusa las limitaciones de la agencia en los planes de desarrollo profesional. Se recalca que es también responsabilidad insustituible de todo buen administrador o administradora, garantizar la existencia de estos planes de desarrollo profesional para todo su personal. 
Profesionales de trabajo social en el rol de administración de agencias de servicio social deben tener preparación para tomar la realidad social de sus respectivos países y convertirla en el plan de trabajo estratégico de su agencia. Asimismo, deben poner en práctica las teorías y diversas estrategias administrativas que le lleven al éxito, ejercer liderazgo con efectividad y visión; ser capaces de transformar la cultura y clima de su agencia para el ofrecimiento de servicios de calidad; transformar a su personal en aprendices, llevar a cabo investigación social, que inventen y renueven las políticas sociales e impulsen la transformación académica del trabajo social en las instituciones universitarias de su país. Los movimientos sociales no sólo demandan recursos, sino también, participación y autodeterminación. Los trabajadores y trabajadoras sociales administrativos trabajando los problemas sociales de sus países, los trabajan también internacionalmente.

Ante el reto, las contribuciones y amenazas de la globalización mundial y su impacto en la administración de agencias, este administrador o administradora debe considerar herramientas de acción para convertirse en el puente entre lo local y lo global. Sus acciones deben incluir: a) el desarrollo de la comunidad como un foco de práctica e incorporar los enfoques de desarrollo de comunidad en toda la práctica de trabajo social; b) desarrollar técnicas para capacitar a personas en desventaja y opresión para que sus voces sean escuchadas en los foros locales, así como internacionales; c) participar activamente en las organizaciones locales y de proyección mundial, como la UNESCO, UNICEF, contribuyendo y promoviendo sus esfuerzos; d) participar en la creación e implantación de programas de intercambio y cursos internacionales; e) desarrollar investigaciones científicas sobre el impacto de los movimientos sociales, particularmente la globalización mundial, sus vínculos entre el conocimiento local y global del trabajo social, sus valores y destrezas; f) participar en convenciones internacionales para que puedan romper el aislamiento activista en la demanda de cambios (Rowe, Handley, Repetur Moreno \& Mould, 2000); g) involucrarse internacionalmente en el manejo de crisis, por ejemplo, en los campos de refugiados de la Naciones Unidas, la Cruz Roja y en actividades de interés común como el Movimiento Internacional de la mujer; y h) participar en el intercambio de trabajo social o iniciativas de adiestramiento internacional, como por ejemplo, entre escuelas de trabajo social o entre profesionales de trabajo social médico-social (Rowe, Handley, Repetur Moreno \& Mould, 2000). Se sugiere además, la creación de asociaciones de profesionales de trabajo social administradores, las cuales, entre otras cosas, 
permitirán: a) reunir a este grupo con experiencias y necesidades similares, bajo un clima de respeto, colaboración y colegialidad, en un proceso compartido de reflexión y evaluación de sus prácticas administrativas; b) desarrollar actividades educativas y sociales que nutran sus conocimientos y competencias administrativas y c) aportar más ampliamente a la solución de los problemas y necesidades de su país.

La globalización ha aumentado los problemas y los profesionales de trabajo social en el rol administrativo enfrentan grandes retos de cambio. Deben entender que este fenómeno presenta unas dificultades que deben contrarrestarse, sin embargo, también presenta oportunidades que el administrador o administradora debe aprovechar.

\section{Referencias}

Caraballo, Ileana, Carriez, Haydeé, Guzmán, Jorge L., Rodríguez, Rosa A., Santiago, Gladys \& Santini, Vilma E. (1992). La gerencia intermedia en los servicios humanos: Conocimientos, funciones y responsabilidades del director local del Departamento de Servicios Sociales de Puerto Rico, agosto 1991 a mayo 1992. Tesis de maestría no publicada, Universidad de Puerto Rico, Río Piedras, Puerto Rico.

Drover, Glen (2000). Redefining social citizenship in a global era. Canada: Ottawa Canadian Association of Social Workers.

Koontz, Harold. \& Weihrich, Heinz (1998). Administración: Una perspectiva global. México, D. F.: McGraw-Hill.

Morales Alejandro, Lourdes I. (2001). Desde la silla del decano. Disertación doctoral no publicada, Universidad de Puerto Rico, Río Piedras, Puerto Rico.

Rowe, William, Hanley, Jill, Repetur Moreno, Eugenia \& Mould, John (2000). Voices of social work practice: International reflections on the effects of globalization. Canada: Ottawa Canadian Association of Social Workers.

Ruíz González, Magali (1999). La práctica de trabajo social: De lo específico a lo genérico. Río Piedras, Puerto Rico: Editorial Edil. 
Skidmore, Rex A. (1995). Social work administration: Dynamic management and human relationship (3ra. Ed.). Massachusetts: Allyn \& Bacon.

Weinbach, Robert W. (2003). The social worker as manager: A practical guide. Boston, MA: Allyn and Bacon. 